\title{
Prognostic factors for pyrrolizidine alkaloid-induced hepatic sinusoidal obstruction syndrome: a multicenter study in China
}

\author{
Haitao Shang ${ }^{1}$, Tao Bai ${ }^{1}$, Shenghua Zhu ${ }^{1}$, Xiaoqian Yang ${ }^{1}$, Chang Liu ${ }^{1}$, Dong Xu ${ }^{2}$, Yuzheng Zhuge ${ }^{3}$, \\ Yuhu Song ${ }^{1}$, Xiaohua Hou ${ }^{1}$ \\ ${ }^{1}$ Division of Gastroenterology, Union Hospital, Tongji Medical College, Huazhong University of Science and Technology, Wuhan, China; \\ ${ }^{2}$ Department of Infectious Diseases, Tongji Hospital, Tongji Medical College, Huazhong University of Science and Technology, Wuhan, China; \\ ${ }^{3}$ Department of Gastroenterology, Drum Tower Hospital, Nanjing University School of Medicine, Nanjing, China \\ Contributions: (I) Conception and design: Y Song, X Hou; (II) Administrative support: Y Song, X Hou; (III) Provision of study materials or patients: \\ D Xu, Y Zhuge, Y Song, X Hou; (IV) Collection and assembly of data: H Shang, S Zhu, X Yang, C Liu; (V) Data analysis and interpretation: H \\ Shang, T Bai, Y Song; (VI) Manuscript writing: All authors; (VII) Final approval of manuscript: All authors. \\ Correspondence to: Xiaohua Hou; Yuhu Song. Division of Gastroenterology, Union Hospital, Tongji Medical College, Huazhong University of Science \\ and Technology, Wuhan 430022, China. Email: houxh@hust.edu.cn; 2009XH0899@hust.edu.cn or yuhusong@yahoo.com.
}

Background: In China, one of the major causes of hepatic sinusoidal obstruction syndrome (HSOS) is the intake of herbals containing pyrrolizidine alkaloid (PA). However, prognostic factors for PA-induced HSOS are poorly understood. The aim of this study was to identify the independent prognostic factors for PAinduced HSOS using a multi-center study.

Methods: A total of 117 PA-induced HSOS patients were enrolled for data collection in three university hospitals from November 2003 to September 2018. Univariate and multivariate Cox proportional hazards analysis were used to determine prognostic factors for PA-induced HSOS.

Results: The median age of the PA-induced HSOS patients was 61 years (range, 21-88 years), and 64\% of them were male. The survival rates at 1 , 3, and 36 months were $89.71 \%, 72.60 \%$, and $69.19 \%$, respectively. Significant differences in prothrombin time (PT), international normalized ratio, total bilirubin, severity grading [new criteria for severity grading of hematopoietic stem cell transplantation (HSCT)-related HSOS in adults] were found between patients who survived and those who died. Univariate and multivariate survival analysis using Cox's regression model demonstrated low serum albumin $(<35 \mathrm{~g} / \mathrm{L})$, elevated serum urea $(>8.2 \mathrm{mmol} / \mathrm{L})$ and severe or very severe HSOS (European Society for Blood and Marrow Transplantation 2016 criteria) were independent prognostic factors of survival.

Conclusions: Serum albumin, serum urea, and severity grading were independent prognostic factors for patients with PA-induced HSOS, and can contribute to identifying potentially high-risk patients for early effective intervention.

Trial registration: ChiCTR-DRD-17010709 (www.chictr.org.cn).

Keywords: Hepatic sinusoidal obstruction syndrome (HSOS); pyrrolizidine alkaloid (PA); prognostic factors

Submitted Jan 14, 2020. Accepted for publication Sep 29, 2020.

doi: $10.21037 /$ atm-20-731

View this article at: http://dx.doi.org/10.21037/atm-20-731

\section{Introduction}

Hepatic sinusoidal obstruction syndrome (HSOS), also known as veno-occlusive disease or VOD, is a kind of hepatic vascular disease which is caused primarily by progenitors of toxic agents to bone marrow and liver sinusoidal endothelial cells (LSECs) $(1,2)$. The major clinical manifestations of HSOS patients are right upperquadrant pain, tender hepatomegaly, ascites, jaundice, and unexplained weight gain. In Western countries, major etiologies of HSOS are cytoreductive therapy prior to 
hematopoietic stem cell transplantation (HSCT), and oxaliplatin-containing chemotherapy (2-4). Meanwhile in China, the major cause of HSOS is the ingestion of herbal medicines containing pyrrolizidine alkaloid (PA) (5-8).

The first case of PA-induced HSOS described by Willmot and Robertson in 1920 was associated with the ingestion of herbal tea containing PA (9). After that, PAinduced HSOS cases have been extensively reported worldwide (10-12). In China, some Chinese herbal medicines contain PAs. Among them, Gynura segetum is widely used, accounting for $50.0-88.6 \%$ of PA-induced HSOS (6). Most of the relevant clinical studies of recent decades have mainly focused on HSCT-related HSOS and oxaliplatin-induced HSOS. Although progress has been made in the pathogenesis, diagnosis, and treatment of PA-induced HSOS in recent years (5,13-17), few studies have investigated the prognostic factors for PAinduced HSOS patients. A recent retrospective study of 117 patients demonstrated that hepatic encephalopathy, and serum bilirubin and albumin levels were major prognostic factors for G. segetum-induced HSOS (18). There were, however, some deficiencies in this study, such as the treatment strategy, follow-up period, and its single-center designs which restrict its scientific value and reliability (18). Consequently, we aimed to conduct a multicenter retrospective study to determine the prognostic factors for PA-induced HSOS patients, in order provide basis for a more informed study of PA-induced HSOS prognosis in the future. This study will help identify high-risk patients at an early stage and help select patients who need immediate therapeutic intervention. We present the following in accordance with the STROBE reporting checklist (available at http://dx.doi.org/10.21037/atm-20-731).

\section{Methods}

\section{Patients selection}

A total of 159 patients with PA-induced HSOS were enrolled in this multi-center retrospective study from November 2003 to September 2018. These patients were admitted to Union Hospital affiliated to Tongji Medical College, Huazhong University of Science and Technology (Wuhan, China); Tongji Hospital affiliated to Tongji Medical College, Huazhong University of Science and Technology (Wuhan, China); or Drum Tower Hospital affiliated to School of Medicine, Nanjing University (Nanjing, China).
The diagnosis of PA-induced HSOS was established according to the Chinese expert consensus (6) which consists of a definite history of PA-containing plant plus pathological evidence or meeting the following three criteria: (A) abdominal distention and/or pain in the hepatic region, hepatomegaly, and ascites; (B) elevation of serum total bilirubin or abnormal liver function testing; (C) typical features of contrast-enhanced computed tomography (CT) or magnetic resonance imaging (MRI). Patients with other liver diseases, such as Budd Chiari syndrome (BCS); viral, alcohol, drug, or autoimmune liver diseases; nonalcoholic fatty liver disease; congestive heart diseases etc. were excluded (6). The other inclusion criteria in the study were the following: (I) patients with PA-induced HSOS; (II) survival patients with a minimum follow-up period of 3 years. Meanwhile, the exclusion criteria were the following: (I) patients who underwent anticoagulation therapy; (II) patients who underwent transjugular intrahepatic portosystemic shunt (TIPS); (III) patients who underwent liver transplantation; (IV) patients lost to followup; (V) survival patients with fewer than 3 years of follow-up (Figure 1). The study was conducted in accordance with the Declaration of Helsinki (as revised in 2013) and approved by institutional ethics committee of Tongji Medical College, Huazhong University of Science and Technology (IORG no: IORG0003571). The study was registered in Chinese Clinical Trial Registry (www.chictr.org.cn, No.: ChiCTRDRD-17010709). A waiver of informed consent was obtained from all patients due to the retrospective nature of the study, and the patient's personal data have been secured.

\section{Data collection}

Clinical profiles and laboratory data were collected at the time of diagnosis. The severity of the PA-induced HSOS patients was graded according to the new criteria of severity grading for HSCT-related HSOS in adults as proposed by the European Society for Blood and Marrow Transplantation (EBMT) (19). All patients were followed up from the date of diagnosis until death, loss to follow-up, or study termination on September 30, 2018.

\section{Statistical analysis}

Continuous variables are represented by median $\left(25^{\text {th }}-75^{\text {th }}\right.$ percentile), and categorical variables are represented by count (percentage). Continuous variables were analyzed with student's test or Mann-Whitney's U test; categorical 


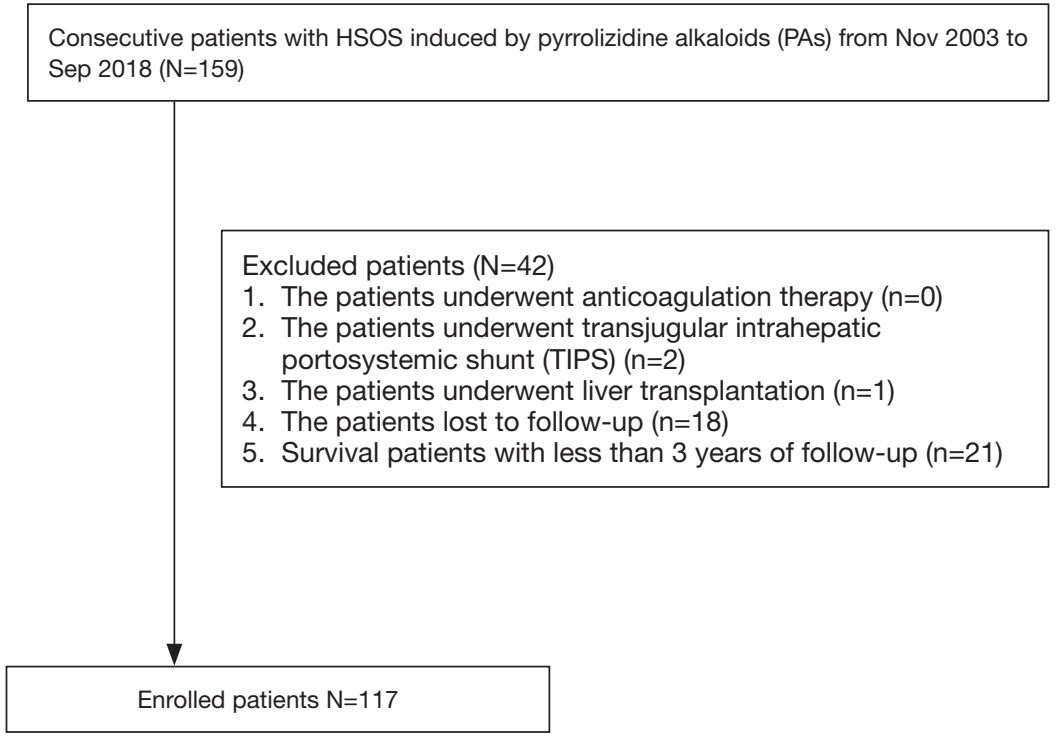

Figure 1 Flowchart showing the inclusion and exclusion criteria for patient selection.

variables were analyzed with Pearson's $\chi^{2}$ or Fisher's exact test. Survival curves were estimated using Kaplan-Meier method, and survival curves were analyzed with the logrank test. Univariate and multivariate analyses were carried out by using Cox proportional hazards regression models to determine independent prognostic factors. All of the significant univariates $(\mathrm{P}<0.1)$ were included in the multivariate analysis. Results are expressed as hazard ratios (HRs) and 95\% confidence intervals (CIs). A P value $<0.05$ was considered statistically significant. All data were statistically analyzed by using SPSS version 19.0 (SPSS Inc., Chicago, IL, USA).

\section{Results}

\section{Baseline clinical and laboratory characteristics}

In this study, 42 cases were excluded and a total of 117 cases met the strict inclusion criteria (Figure 1). The characteristics of patients are shown in Table 1. Overall, the median age of the patients was 61 years old (range, $21-88$ years) and $64 \%(75 / 117)$ of these patients were male (Table 1). Firstly, the results of blood routine examination revealed that the median values of erythrocyte, leukocyte, hemoglobin, and platelet were all within the normal range. Secondly, the elevation of liver enzymes [aspartate transaminase (AST) and alanine aminotransferase (ALT)] and total bilirubin was observed in PA-induced HSOS patients, and the abnormality of albumin and prothrombin time (PT) in PA-induced HSOS patients indicated the damaged synthetic ability of liver (Table 1). Finally, renal function was normal in the most patients, which was revealed by serum urea and creatinine.

Baseline clinical characteristics of the two groups (survival group and death group) were measured and summarized (Table 1). Significant differences existed in PT (15.90 vs. $17.00 \mathrm{~s}, \mathrm{P}=0.03)$, international normalized ratio (1.29 vs. 1.42, $\mathrm{P}=0.02)$, total bilirubin $(34.31$ vs. $43.95 \mu \mathrm{mol} / \mathrm{L}$, $\mathrm{P}=0.03$ ) between the survival group and the death group. The Child-Pugh score (CPS) and model for end-stage liver disease (MELD) score are widely used as tools to assess the prognosis of liver cirrhosis. As expected, ChildPugh and MELD scores were significantly higher in the death group than those in the survival group. Recently, the EMBT proposed a new HSCT-related HSOS severity classification system for adults (2016) (19). The severe or extreme mortality rate $(61.11 \%)$ was higher than the mild or moderate mortality rate (38.89\%).

\section{Survival and prognostic factors}

The median follow-up time for all patients was 1,159 days (interquartile range 77.5-1,988). At last follow-up, 81 $(69.23 \%)$ of the 117 patients were alive. The 1-, 3-, and 36-month survival rates were $89.71 \%$ [95\% confidence interval (CI), 84.21-95.19\%], 72.60\% (95\% CI, $64.57-$ $80.64 \%$ ), and $69.19 \%$ (95\% CI, 60.78-77.63\%), respectively 
Table 1 Baseline characteristics of 117 patients with PA-induced HSOS at the time of diagnosis

\begin{tabular}{|c|c|c|c|c|}
\hline Variables & All patients & \multicolumn{3}{|c|}{ Survival outcome } \\
\hline Number of patients & 117 & 81 & 36 & \\
\hline Age, years & $61.00(50.50-64.50)$ & $60.00(49.50-64.50)$ & $61.00(51.25-64.75)$ & 0.89 \\
\hline Male sex, n (\%) & $75.00(64.00 \%)$ & $50.00(61.70 \%)$ & $25.00(69.40 \%)$ & 0.42 \\
\hline Hemoglobin, g/L & $139.00(122.50-150.00)$ & $137.50(122.25-149.00)$ & $141.00(121.50-159.00)$ & 0.32 \\
\hline Leukocyte, $10^{9} / \mathrm{L}$ & $6.08(4.66-8.21)$ & $5.74(4.44-7.19)$ & $7.51(5.30-8.73)$ & 0.20 \\
\hline Platelet, $10^{9} / \mathrm{L}$ & $104.00(75.00-142.00)$ & $106.00(75.50-155.50)$ & $98.00(72.00-128.00)$ & 0.31 \\
\hline PT, S & $16.60(15.08-18.38)$ & $15.90(14.90-18.10)$ & $17.00(16.20-18.70)$ & 0.03 \\
\hline AST, U/L & $91.00(53.00-165.00)$ & $79.00(48.00-162.00)$ & $111.00(67.25-188.00)$ & 0.23 \\
\hline Total bilirubin, $\mu \mathrm{mol} / \mathrm{L}$ & 36.35 (22.85-67.35) & $34.31(21.71-56.32)$ & 43.95 (26.75-116.90) & 0.03 \\
\hline ALB, g/L & $31.55(28.30-35.20)$ & $32.85(28.80-35.58)$ & $29.85(27.25-32.53)$ & 0.18 \\
\hline Urea, mmol/L & $5.95(4.36-8.18)$ & $5.97(4.48-7.62)$ & $5.86(4.22-8.96)$ & 0.28 \\
\hline Creatinine, $\mu \mathrm{mol} / \mathrm{L}$ & 77.50 (61.07-93.08) & 75.50 (62.20-91.70) & 81.95 (62.08-99.40) & 0.14 \\
\hline Child-Pugh, n (\%) & & & & 0.08 \\
\hline A & $19.00(17.12)$ & $17.00(22.37)$ & $2.00(5.71)$ & \\
\hline B & $75.00(67.57)$ & $49.00(64.47)$ & $26.00(74.29)$ & \\
\hline Very severe & $23.00(19.83)$ & $11.00(13.75)$ & $12.00(33.33)$ & \\
\hline MELD & 11.32 (8.13-15.63) & $10.12(6.60-14.84)$ & 15.28 (9.69-17.29) & $<0.01$ \\
\hline
\end{tabular}

Continuous variables are presented as median $\left(25^{\text {th }}-75^{\text {th }}\right.$ percentiles), and categorical variables are presented as count (percentage).

*, the $\mathrm{P}$ values refer to $t$-test or chi-square test between patients in the survival group and death group; ${ }^{\dagger}$, refers to the new criteria for severity grading of HSCT-related HSOS in adults (19). Normal ranges: erythrocytes: (3.0-5.5)×10 12/L; hemoglobin: 110-160 g/L; leukocyte: $(4.0-10.0) \times 10^{9} / \mathrm{L}$; platelet: $(100.0-300.0) \times 10^{9} / \mathrm{L}$; prothrombin time (PT): 11-16 S; international normalized ratio (INR): $0.80-1.31$; alanine aminotransferase (ALT): 5-35 U/L; aspartate aminotransferase (AST): 8-40 U/L; total bilirubin (T-BIL): 5.1-19 $\mu \mathrm{mol} / \mathrm{L} ;$ albumin (ALB): 35-55 g/L; urea: 2.9-8.2 mmol/L; creatinine: 44-106 $\mu \mathrm{mol} / \mathrm{L}$. PA, pyrrolizidine alkaloid; HSOS, hepatic sinusoidal obstruction syndrome; MELD, model for end-stage liver disease. 

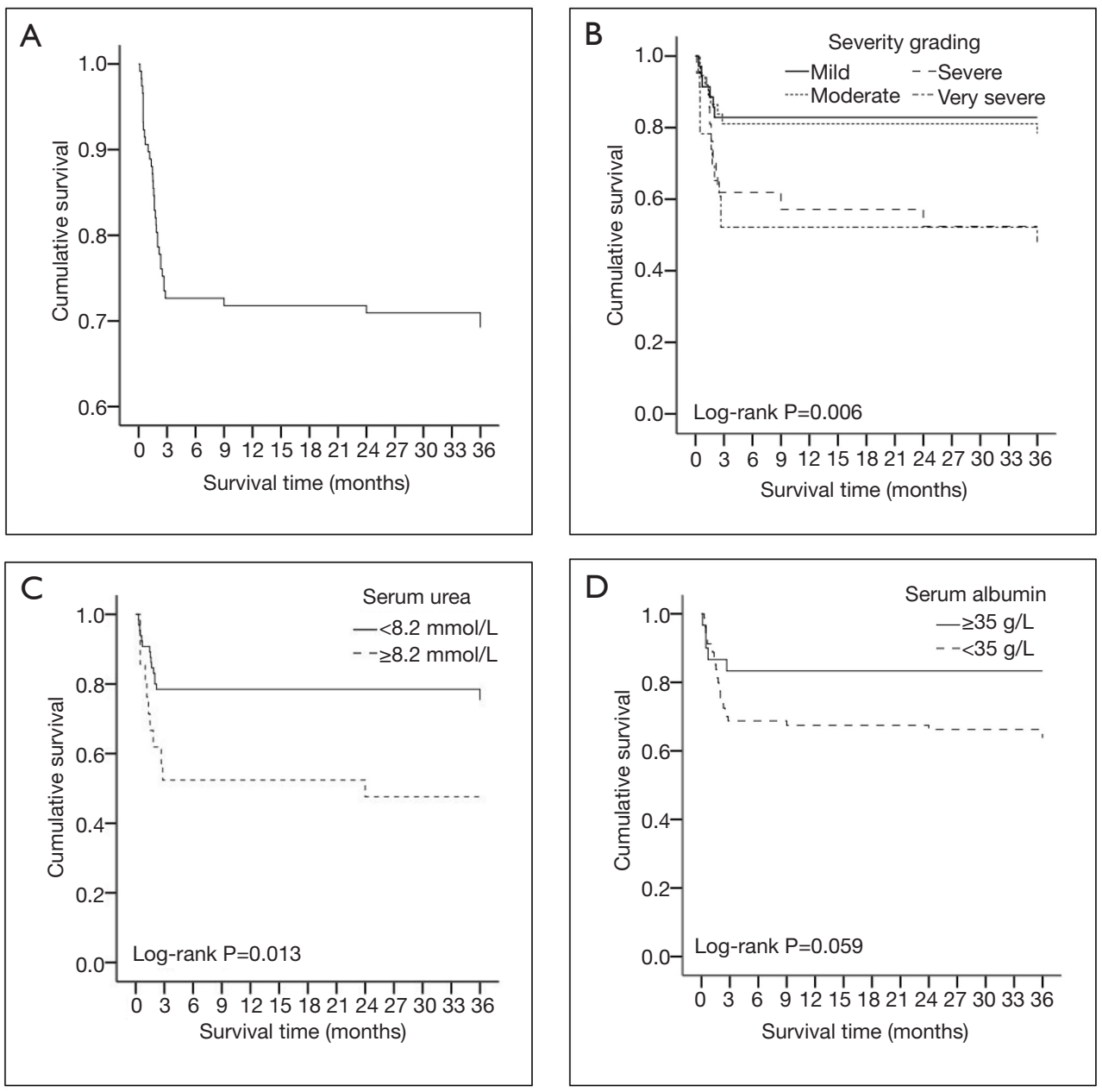

Figure 2 Survival curves in the 117 cases of HSOS induced by Gynura segetum. (A) Overall survival of enrolled patients; (B) survival of patients according to severity grading; (C) survival of patients according to serum urea; (D) survival of patients according to serum albumin. HSOS, hepatic sinusoidal obstruction syndrome.

(Figure 2A). Univariate Cox proportional hazards analysis was then used to determine predictors of survival for PA-induced HSOS. Univariate analysis showed that 10 independent variables were significantly associated with a poor survival rate $(\mathrm{P}<0.1)$ : total bilirubin $>51.30 \mu \mathrm{mol} / \mathrm{L}$, AST level $>2$ upper limit of normal (ULN), serum albumin $<35.00 \mathrm{~g} / \mathrm{L}$, international normalized ratio (INR) level $>1$ ULN, PT $>1$ ULN, serum urea $>1$ ULN, Fibrosis 4 Score (FIB-4) score $>3.25$, Child-Pugh class B or C, MELD score $>14$, and severe-to-very severe patients (19) (EBMT 2016 criteria) (Table 2). Importantly, a multivariate analysis of prognostic factors was performed. The results showed that serum albumin level, serum urea level, and severity grading were independent prognostic factors for the survival of the 117 PA-induced HSOS patients.

We then analyzed the survival curve of PA-induced HSOS patients. The 3 -year overall survival rate of severe or extreme severe patients was $63.00 \%$, and the survival rate of mild or moderate patients was $83.32 \%$ (Figure $2 B$ ). In addition, the 3 -year overall survival rates of patients with high serum urea $(\geq 8.2 \mu \mathrm{mol} / \mathrm{L})$ and low serum urea $(<8.2 \mu \mathrm{mol} / \mathrm{L})$ were $47.63 \%$ and $75.39 \%$, respectively (Figure 2C). At 3 years, the cumulative survival rates of the patients with normal serum albumin level $(\geq 35 \mathrm{~g} / \mathrm{L})$ and low serum albumin level $(<35 \mathrm{~g} / \mathrm{L})$ were $83.91 \%$ and $63.82 \%$, respectively (Figure $2 D$ ). Unfortunately, the $\mathrm{P}$ value between 
Table 2 Univariate and multivariate analysis of prognostic factors for PA-induced HSOS

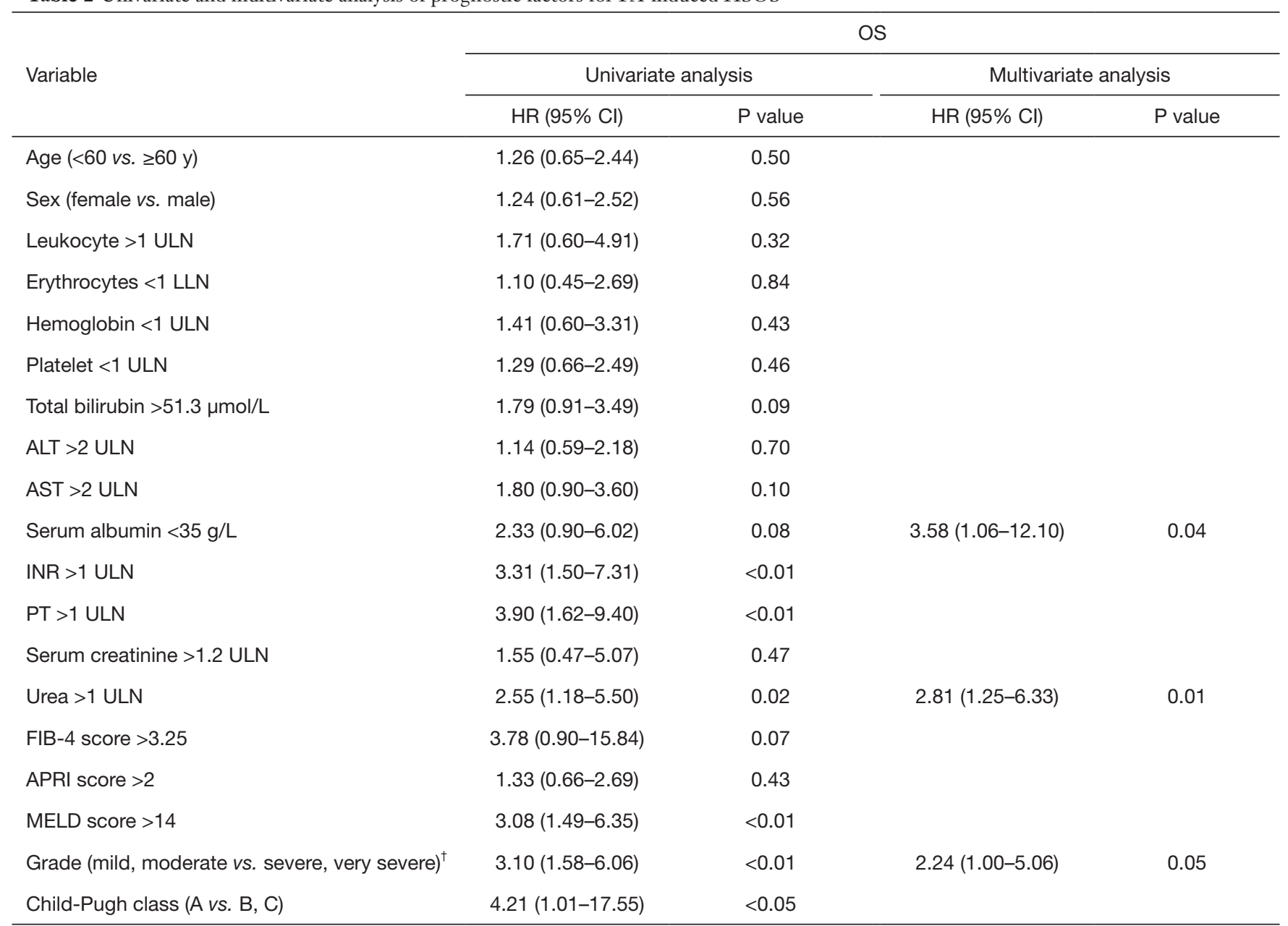

$\mathrm{HR}$ and relative $\mathrm{P}$ values were estimated by Cox proportional hazard regression analysis. ${ }^{\dagger}$, refers to the new criteria for severity grading of HSCT-related HSOS in adults (19). PA, pyrrolizidine alkaloid; HSOS, hepatic sinusoidal obstruction syndrome; OS, overall survival; HR, hazard ratio; CI, confidence interval; ULN, upper limit of normal; LLN, lower limits of normal; APRI, AST-to Platelet Ratio; FIB-4, Fibrosis 4 Score; MELD, model for end-stage liver disease.

these two groups was $0.06(\mathrm{P}=0.06)$ with this unimpressive p-value probably resulting from the small sample size (Figure 2D).

\section{Discussion}

In China, one of the major causes of HSOS is the intake of herbals containing PA. However, prognostic factors for PA-induced HSOS are poorly understood. Due to the differences in etiology, the outcome of patients with HSCTrelated HSOS cannot be extrapolated to that of patients with PA-induced HSOS. In our retrospective multi-center study, clinical profiles, laboratory data, and outcomes were collected. The results demonstrated that serum albumin, serum urea, and severity grading (19) (EBMT 2016 criteria) were independent prognostic factors for PA-induced HSOS, and these findings may contribute to identifying potentially high-risk patients for early effective intervention.

Firstly, serum albumin is a marker of liver synthetic function, and was demonstrated to be an independent prognostic factor. Secondly, serum urea was identified as an independent prognostic factor. To ensure its reliability, the patients with chronic kidney disease and drug-induce renal injury were excluded. In PA-induced HSOS, the damage of sinusoidal endothelial cells and the obstruction of sinusoidal venous outflow result in post-sinusoidal portal hypertension. Then, ascites and edema caused by portal hypertension lead to a reduction in effective circulating blood volume, 
resulting in the elevation of serum urea. Thirdly, it is well established that the new EBMT severity-grading criteria (EBMT 2016 criteria) for HSCT-related HSOS includes the time of first clinical symptoms, bilirubin, transaminase, weight increase, and renal function (19). The results showed that severity grading (EBMT 2016 criteria) was positively correlated with PA-induced HSOS results. With further analysis, it was found that severity grading is an independent prognostic factor. However, the results suggested that the level of bilirubin, transaminase, and serum creatinine were not independent prognostic factors. This means that the new EBMT severity-grading criteria used for HSCT-related HSOS does not necessarily apply to the severity-grading system of PA-induced HSOS. In summary, a new severity grading system for PA-induced HSOS should be established and validated in prospective studies.

To the best of our knowledge, our is the first multicenter study to examine the prognostic factors for the PA-induced HSOS patients. In this study, the 3-year survival rate was $69.19 \%$, which was higher than that of the patients with HSCT-related HSOS (20-24). The difference in survival time between HSCT-related HSOS and PA-induced HSOS is associated with underlying disease and the function of bone marrow-derived endothelial progenitor cells. In the related research, adult patients undergoing HSCT usually have been found with hematological malignancy; in addition, HSCT-related HSOS is prone to develop into severe status since cytoreductive therapy prior to HSCT damages bone marrow-derived endothelial progenitor cells which replenish the injured sinusoidal endothelial cells (25-27). In addition, the pathogenesis, clinical profiles, and pathological features vary with the different causes of HSOS. Sustained and uncontrolled endothelial/sinusoidal activation is the likely culprit of thrombocytopenia in patients with HSCTrelated HSOS, and thus thrombocytopenia was listed in the 2017 EBMT diagnostic criteria for children (28). Thrombocytopenia have not been observed in most PAinduced HSOS and oxaliplatin-induced HSOS (5,18,29-31). Interestingly, platelet aggregation in Disse's space is involved in the development of SOS $(32,33)$, and thus the inhibition of platelet aggregation with phosphodiesterase III inhibitors can attenuate rat sinusoidal obstruction syndrome (34). In the pathogenesis of HSOS, LSECs are damaged by toxic metabolites of PAs, pyrroleprotein adducts (PPAs) in PA-induced HSOS (35); while toxic damage of LSECs may result from increased metabolic activation by LSECs (dacarbazine) or decreased detoxification by glutathione (GSH) (azathioprine and the cyclophosphamide metabolite, acrolein). In pathological examination, massive sinusoidal congestion and hepatocellular necrosis predominantly in zone 3 have been found to be pathological features of PA-induced HSOS and HSCT-related HSOS; multifocal sinusoidal dilatation without hepatocytes necrosis has also been observed in oxaliplatin-induced HSOS (30,36).

To our knowledge, several studies have described the outcome of PA-induced HSOS patients. A systematic review of published case reports showed that increased total bilirubin and AST were indicators of poor survival in patients with PA-induced HSOS (37). Gao et al.'s study demonstrated that the concentration of blood PPAs was related to the severity and clinical outcome of PA-induced HSOS (38); however, the assay of PPAs concentration by ultra-performance liquid chromatography-tandem mass spectrometry (UPLC-M) analysis was not available in most of hospitals. In addition, the concentration of PPA in the blood decreased significantly after 40 days of exposure to PA. It is clear that histological evidence plays a critical role in evaluating prognosis. Unfortunately, only 12 patients received liver biopsy due to ascites, thrombocytopenia, and coagulation disorders in this study. Fortunately, a diagnosis of PA-HSOS can also be established by diagnostic criteria consisting of history, clinical manifestations, lab examinations, and imaging signs (Figure 3) (6). Sinusoidal dilation and congestion, the necrosis of hepatocytes, the extravasation of erythrocytes in zone 3 were found to be the characteristics of PA-induced HSOS (Figure 3) $(39,40)$.

Recently, a retrospective study reported 117 cases of hepatic encephalopathy, and serum bilirubin and albumin levels were found to be major prognostic factors affecting $G$. segetum-induced HSOS (18). However, several shortcomings of the study restrict its value. Firstly, it was a retrospective, single-center study. Secondly, enrolled patients received different therapeutic approaches including anticoagulant therapy or TIPS, and anticoagulant therapy or TIPS probably improved the outcome of the patients (6). Thirdly, the follow-up period ranged from 3 days to 60 months (18), and survival patients with a short follow-up period should be excluded. Finally, clinical characteristics of the patients who received non-anticoagulant therapy or anticoagulant therapy should be similar; however, relevant data of the patients were not provided. These factors limited the scientific value and reliability of the research. Therefore, our study used stringent inclusion criteria such as similar therapeutic strategy, long-term follow-up periods, and 

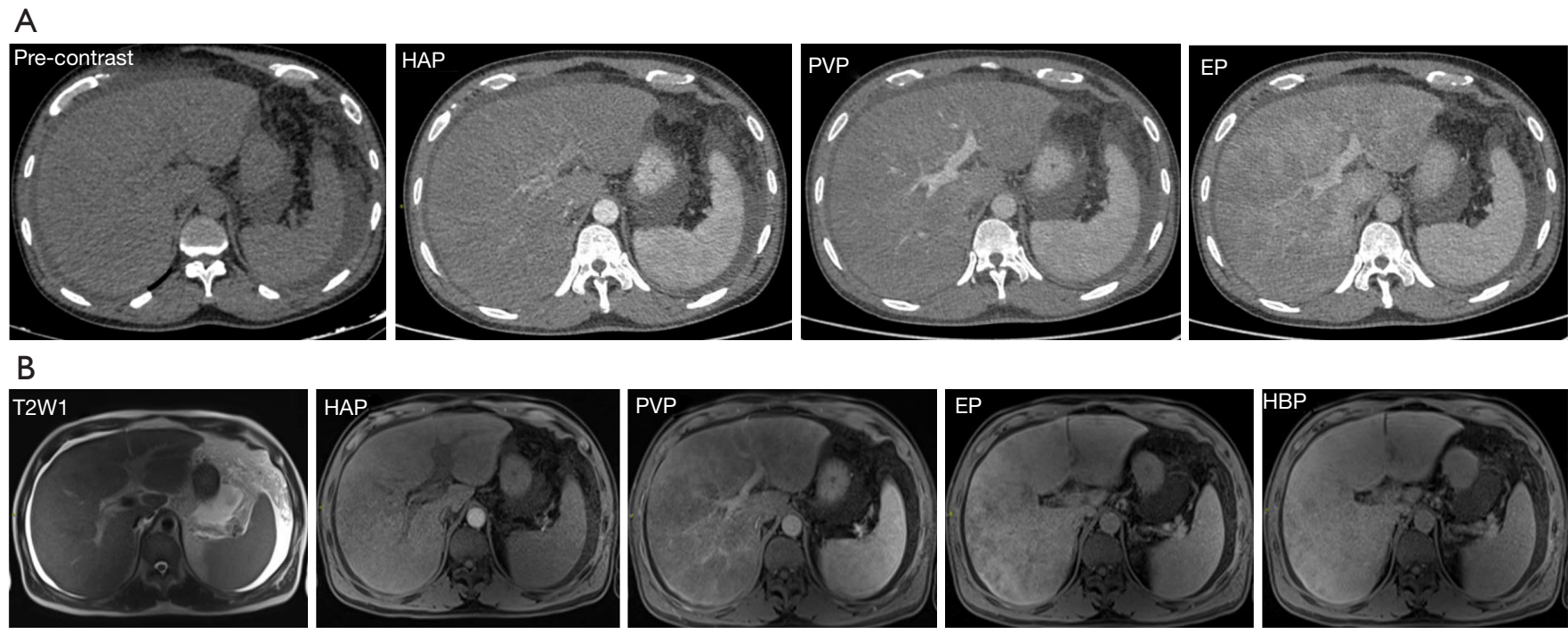

C
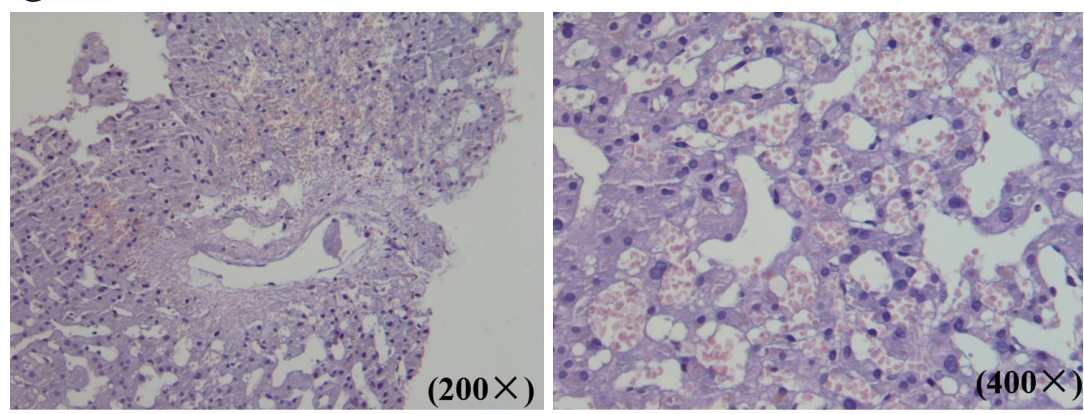

Figure 3 Representative CT, MRI, and histological imaging. (A) Representative CT image. (B) Representative MRI image; 49-year-old man with Gynura segetum-induced HSOS received contrast-enhanced CT and a gadoxetic acid-enhanced MRI scan. Heterogeneous hypoattenuation/hypointensity and patchy enhancement are shown. (C) Representative histological results; 36-year-old man with Gynura segetum-induced HSOS received liver biopsy, with the results showing massive sinusoidal dilatation and sinusoidal congestion accompanied by the extravasation of erythrocytes into the space of Disse. HAP, hepatic arterial phase; PVP, porta-venous phase; EP, equilibrium phase; HBP, hepatobiliary phase; HSOS, hepatic sinusoidal obstruction syndrome.

multi-center participation which to improve the reliability.

These measures notwithstanding, our study had several limitations. Firstly, it was a retrospective cohort study, and unrecognized biases could not be excluded. Secondly, enrolled patients were recruited from 2003 to 2018, and the consensus guidelines on clinical management of PA-induced HSOS were published by the Hepatobiliary Diseases Committee of the Chinese Society of Gastroenterology in 2018 (6). Thus, therapeutic strategies for PA-induced HSOS were based on respective institutional protocols and physicians' decisions in this study, and the outcomes might have been overestimated or underestimated. Thirdly, the efficacy of anticoagulant, TIPS and liver transplantation has been demonstrated in PA-induced HSOS patients; thus, the patients who received the treatment of anticoagulant, TIPS, and liver transplantation were excluded in the study in order to guarantee the reliability of our study. Fourthly, the correlation between the dosage of PA exposure and the prognosis was not determined. In China, plants containing $\mathrm{PA}$ are soaked in liquid, and the liquid containing $\mathrm{PA}$ is ingested by patients; given this, it was difficult to precisely calculate the dosage of PA exposure. Finally, as only $10 \%$ $(12 / 117)$ of the PA-induced HSOS patients underwent liver biopsy, the correlation between lesion and patient prognosis could not be determined.

\section{Conclusions}


We identified three important prognostic factors for PAinduced HSOS: serum albumin, serum urea, and severity grading, which can aid in identifying high-risk patients who should receive early clinical intervention. Prospective studies will be performed to validate the results, the findings of which can act as a basis for a new severity-grading system for PA-induced HSOS.

\section{Acknowledgments}

We would like to thank AME Editing Service and Zhongshan U-YES Translation Service Co, Ltd, for providing language editing.

Funding: This work was partially supported by the National Natural Science Foundation of China (No. 81570555).

\section{Footnote}

Reporting Checklist: The authors have completed the STROBE reporting checklist (available at http://dx.doi. org/10.21037/atm-20-731)

Data Sharing Statement: Available at http://dx.doi. org/10.21037/atm-20-731

Conflicts of Interest: All authors have completed the ICMJE uniform disclosure form (available at http://dx.doi. org/10.21037/atm-20-731). The authors have no conflicts of interest to declare.

Ethical Statement: The authors are accountable for all aspects of the work in ensuring that questions related to the accuracy or integrity of any part of the work are appropriately investigated and resolved. The study was conducted in accordance with the Declaration of Helsinki (as revised in 2013) and approved by institutional ethics committee of Tongji Medical College, Huazhong University of Science and Technology (IORG no: IORG0003571). The study was registered in Chinese Clinical Trial Registry (www.chictr.org.cn, No.: ChiCTR-DRD-17010709). A waiver of informed consent was obtained from all patients due to the retrospective nature of the study, and the patient's personal data have been secured.

Open Access Statement: This is an Open Access article distributed in accordance with the Creative Commons Attribution-NonCommercial-NoDerivs 4.0 International License (CC BY-NC-ND 4.0), which permits the non- commercial replication and distribution of the article with the strict proviso that no changes or edits are made and the original work is properly cited (including links to both the formal publication through the relevant DOI and the license). See: https://creativecommons.org/licenses/by-nc-nd/4.0/.

\section{References}

1. Valla DC, Cazals-Hatem D. Sinusoidal obstruction syndrome. Clin Res Hepatol Gastroenterol 2016;40:378-85.

2. DeLeve LD, Valla DC, Garcia-Tsao G. Vascular disorders of the liver. Hepatology 2009;49:1729-64.

3. Dignan FL, Wynn RF, Hadzic N, et al. BCSH/BSBMT guideline: diagnosis and management of veno-occlusive disease (sinusoidal obstruction syndrome) following haematopoietic stem cell transplantation. Br J Haematol 2013;163:444-57.

4. Bayraktar UD, Seren S, Bayraktar Y. Hepatic venous outflow obstruction: three similar syndromes. World J Gastroenterol 2007;13:1912-27.

5. Kan X, Ye J, Rong X, et al. Diagnostic performance of Contrast-enhanced CT in Pyrrolizidine Alkaloids-induced Hepatic Sinusoidal Obstructive Syndrome. Sci Rep 2016;6:37998.

6. Zhuge Y, Liu Y, Xie W, et al. Expert consensus on the clinical management of pyrrolizidine alkaloid-induced hepatic sinusoidal obstruction syndrome. J Gastroenterol Hepatol 2019;34:634-42.

7. Lin G, Wang JY, Li N, et al. Hepatic sinusoidal obstruction syndrome associated with consumption of Gynura segetum. J Hepatol 2011;54:666-73.

8. Wang JY, Gao H. Tusanqi and hepatic sinusoidal obstruction syndrome. J Dig Dis 2014;15:105-7.

9. Willmot FC, Robertson GW. Senecio disease or cirrhosis of the liver due to Senecio poisoning. Lancet 1920;196:848-9.

10. Kakar F, Akbarian Z, Leslie T, et al. An outbreak of hepatic veno-occlusive disease in Western afghanistan associated with exposure to wheat flour contaminated with pyrrolizidine alkaloids. J Toxicol 2010;2010:313280.

11. Dai N, Yu YC, Ren TH, et al. Gynura root induces hepatic veno-occlusive disease: a case report and review of the literature. World J Gastroenterol 2007;13:1628-31.

12. Chojkier M. Hepatic sinusoidal-obstruction syndrome: toxicity of pyrrolizidine alkaloids. J Hepatol 2003;39:437-46.

13. Guo T, Li X, Yang X, et al. Gadoxetic Acid-Enhanced Hepatobiliary-Phase Magnetic Resonance Imaging 
for Pyrrolizidine Alkaloid-Induced Hepatic Sinusoidal Obstruction Syndrome and Association with Liver Function. Sci Rep 2019;9:1231.

14. Li X, Yang X, Xu D, et al. Magnetic Resonance Imaging Findings in Patients With Pyrrolizidine AlkaloidInduced Hepatic Sinusoidal Obstruction Syndrome. Clin Gastroenterol Hepatol 2017;15:955-7.

15. Wu XW, Wang WQ, Liu B, et al. Hepatic veno-occlusive disease after taking Gynura Rhizome: The value of multidetector computed tomography in diagnosing the disease and evaluating the clinical therapeutic effect. Hepatol Res 2012;42:304-9.

16. Zhuge YZ, Wang Y, Zhang F, et al. Clinical characteristics and treatment of pyrrolizidine alkaloid-related hepatic vein occlusive disease. Liver Int 2018;38:1867-74.

17. Yang XQ, Ye J, Li X, et al. Pyrrolizidine alkaloids-induced hepatic sinusoidal obstruction syndrome: Pathogenesis, clinical manifestations, diagnosis, treatment, and outcomes. World J Gastroenterol 2019;25:3753-63.

18. Wang Y, Qiao D, Li Y, et al. Risk factors for hepatic veno-occlusive disease caused by Gynura segetum: a retrospective study. BMC Gastroenterol 2018;18:156.

19. Mohty M, Malard F, Abecassis M, et al. Revised diagnosis and severity criteria for sinusoidal obstruction syndrome/veno-occlusive disease in adult patients: a new classification from the European Society for Blood and Marrow Transplantation. Bone Marrow Transplant 2016;51:906-12.

20. Dalle JH, Giralt SA. Hepatic Veno-Occlusive Disease after Hematopoietic Stem Cell Transplantation: Risk Factors and Stratification, Prophylaxis, and Treatment. Biol Blood Marrow Transplant 2016;22:400-9.

21. Corbacioglu S, Carreras E, Mohty M, et al. Defibrotide for the Treatment of Hepatic Veno-Occlusive Disease: Final Results From the International Compassionate-Use Program. Biol Blood Marrow Transplant 2016;22:1874-82.

22. Richardson PG, Riches ML, Kernan NA, et al. Phase 3 trial of defibrotide for the treatment of severe venoocclusive disease and multi-organ failure. Blood 2016;127:1656-65.

23. Richardson PG, Smith AR, Triplett BM, et al. Defibrotide for Patients with Hepatic Veno-Occlusive Disease/ Sinusoidal Obstruction Syndrome: Interim Results from a Treatment IND Study. Biol Blood Marrow Transplant 2017;23:997-1004.

24. Yakushijin K, Atsuta Y, Doki N, et al. Sinusoidal obstruction syndrome after allogeneic hematopoietic stem cell transplantation: Incidence, risk factors and outcomes.
Bone Marrow Transplant 2016;51:403-9.

25. Harb R, Xie G, Lutzko C, et al. Bone marrow progenitor cells repair rat hepatic sinusoidal endothelial cells after liver injury. Gastroenterology 2009;137:704-12.

26. Wang L, Wang X, Xie G, et al. Liver sinusoidal endothelial cell progenitor cells promote liver regeneration in rats. J Clin Invest 2012;122:1567-73.

27. Wang L, Wang X, Wang L, et al. Hepatic vascular endothelial growth factor regulates recruitment of rat liver sinusoidal endothelial cell progenitor cells. Gastroenterology 2012;143:1555-63.e2.

28. Corbacioglu S, Carreras E, Ansari M, et al. Diagnosis and severity criteria for sinusoidal obstruction syndrome/venoocclusive disease in pediatric patients: a new classification from the European society for blood and marrow transplantation. Bone Marrow Transplant 2018;53:138-45.

29. Zhuge YZ, Wang Y, Zhang F, et al. Clinical characteristics and treatment of pyrrolizidine alkaloid-related hepatic vein occlusive disease. Liver Int 2018;38:1867-74.

30. Liu F, Cao X, Ye J, et al. Oxaliplatin-induced hepatic sinusoidal obstruction syndrome in a patient with gastric cancer: A case report. Mol Clin Oncol 2018;8:453-6.

31. Han NY, Park BJ, Kim MJ, et al. Hepatic Parenchymal Heterogeneity on Contrast-enhanced CT Scans Following Oxaliplatin-based Chemotherapy: Natural History and Association with Clinical Evidence of Sinusoidal Obstruction Syndrome. Radiology 2015;276:766-74.

32. Hirata M, Tajima H, Miyashita T, et al. Extravasated platelet aggregation in the livers of rats with druginduced hepatic sinusoidal obstruction syndrome. Mol Med Rep 2017;15:3147-52.

33. Nakanuma S, Miyashita T, Hayashi H, et al. Extravasated platelet aggregation in liver zone 3 may correlate with the progression of sinusoidal obstruction syndrome following living donor liver transplantation: A case report. Exp Ther Med 2015;9:1119-24.

34. Miyata T, Tajima H, Hirata M, et al. Phosphodiesterase III inhibitor attenuates rat sinusoidal obstruction syndrome through inhibition of platelet aggregation in Disse's space. J Gastroenterol Hepatol 2018;33:950-7.

35. Yan CC, Huxtable RJ. Quantitation of the hepatic release of metabolites of the pyrrolizidine alkaloid, monocrotaline. Toxicol Appl Pharmacol 1994;127:58-63.

36. Ward J, Guthrie JA, Sheridan MB, et al. Sinusoidal obstructive syndrome diagnosed with superparamagnetic iron oxide-enhanced magnetic resonance imaging in patients with chemotherapy-treated colorectal liver metastases. J Clin Oncol 2008;26:4304-10. 
37. Wang X, Qi X, Guo X. Tusanqi-Related Sinusoidal Obstruction Syndrome in China: A Systematic Review of the Literatures. Medicine (Baltimore) 2015;94:e942.

38. Gao H, Ruan JQ, Chen J, et al. Blood pyrroleprotein adducts as a diagnostic and prognostic index in pyrrolizidine alkaloid-hepatic sinusoidal obstruction syndrome. Drug Des Devel Ther 2015;9:4861-8.

Cite this article as: Shang $H$, Bai $T$, Zhu S, Yang $X$, Liu C, Xu D, Zhuge Y, Song Y, Hou X. Prognostic factors for pyrrolizidine alkaloid-induced hepatic sinusoidal obstruction syndrome: a multicenter study in China. Ann Transl Med 2021;9(1):11. doi: 10.21037/atm-20-731
39. Fan CQ, Crawford JM. Sinusoidal obstruction syndrome (hepatic veno-occlusive disease). J Clin Exp Hepatol 2014;4:332-46.

40. Liu F, Rong X, Guo H, et al. Clinical characteristics, CT signs, and pathological findings of Pyrrolizidine alkaloidsinduced sinusoidal obstructive syndrome: a retrospective study. BMC Gastroenterol 2020;20:30. 